\title{
THE ASSEMBLY HISTORY OF DISK GALAXIES. II. PROBING THE EMERGING TULLY-FISHER RELATION DURING $1<z<1.7$
}

\author{
Sarah H. Miller ${ }^{1,2}$, Richard S. Ellis ${ }^{2}$, Mark Sullivan $^{1}$, Kevin Bundy $^{3}$, Andrew B. Newman $^{2}$, And Tommaso Treu $^{4}$ \\ ${ }^{1}$ Oxford Astrophysics, University of Oxford, Oxford OX1 3RH, UK; s.miller1@ physics.ox.ac.uk \\ ${ }^{2}$ Cahill Center for Astronomy and Astrophysics, California Institute of Technology, Pasadena, CA 91125, USA \\ ${ }^{3}$ Institute for the Physics and Mathematics of the Universe (IPMU), University of Tokyo, Japan \\ ${ }^{4}$ Physics Department, University of California, Santa Barbara, CA 93106, USA \\ Received 2012 January 19; accepted 2012 April 30; published 2012 June 14
}

\begin{abstract}
Through extended integrations using the recently installed deep depletion CCD on the red arm of the Keck I Low Resolution Imaging Spectrograph, we present new measurements of the resolved spectra of 70 morphologically selected star-forming galaxies with $i_{\mathrm{AB}}<24.1$ in the redshift range $1 \lesssim z<1.7$. Using the formalism introduced in Paper I of this series and available Hubble Space Telescope (HST) Advanced Camera for Surveys images, we successfully recover rotation curves using the extended emission line distribution of [O II] $3727 \AA$ to 2.2 times the disk scale radius for a sample of 42 galaxies. Combining these measures with stellar masses derived from $H S T$ and ground-based near-infrared photometry enables us to construct the stellar mass Tully-Fisher relation $\left(M_{*}\right.$-TFR) in the time interval between the well-constructed relation defined at $z \simeq 1$ in Paper I and the growing body of resolved dynamics probed with integral field unit spectrographs at $z>2$. Remarkably, we find a well-defined TFR with up to $60 \%$ increase in scatter and zero-point shift constraint of $\Delta M_{*}=0.02 \pm 0.02$ dex since $z \sim 1.7$, compared to the local relation. Although our sample is incomplete in terms of either a fixed stellar mass or star formation rate limit, we discuss the implications that typical star-forming disk galaxies evolve to arrive on a well-defined TFR within a surprisingly short period of cosmic history.
\end{abstract}

Key words: galaxies: evolution - galaxies: fundamental parameters - galaxies: kinematics and dynamics galaxies: spiral

Online-only material: color figures

\section{INTRODUCTION}

Excellent progress has been made over the past 15 years in observationally charting the history of cosmic star formation (Hopkins \& Beacom 2006; Ellis 2008). Attention has now moved to interpreting the stellar mass assembly history of galaxies (e.g., Bundy et al. 2006) in a framework whereby cold dark matter halos merge under gravitational instability (White \& Rees 1978). Combining dynamical and stellar masses for selected galaxies at various look-back times provides insight into the relative contributions of dark and baryonic matter through time. For the most abundant population of star-forming galaxies, the Tully-Fisher relation (TFR), which probes the relationship between luminosity and rotational velocity, is the most effective tool in assessing the angular momentum development of disks as they assemble in dark matter halos (Fall \& Efstathiou 1980; Blumenthal et al. 1984; Mo et al. 1998; Navarro \& Steinmetz 2000). Of particular value is the stellar mass Tully-Fisher relation $\left(M_{*}\right.$-TFR), which is tighter than any scaling relation confined to a given luminosity band (Bell \& de Jong 2001) making it highly sensitive to self-similar disk growth through time.

In Paper I of this series (Miller et al. 2011) we demonstrated, using extended integrating times with DEIMOS on the Keck 2 telescope together with an optimal technique for extracting kinematic rotation curves to a location corresponding to 2.2 disk scale radii $\left(2.2 r_{s}\right.$ or $\left.r_{2.2}\right)$, a $M^{*}$-TFR at $z \simeq 1$ that has virtually the same intrinsic scatter as that observed locally. This indicates that most disk galaxies at LMC mass or higher are dynamically mature by $z \simeq 1$, in contrast to the conclusions of earlier studies based on shorter exposure times or other velocity extraction methods (Conselice et al. 2005; Flores et al. 2006; Kassin et al. 2007). Moreover, the zero-point shift in the relationship from $z \simeq 1$ to $z \simeq 0.2$ is marginal $\left(\Delta M_{*} \sim 0.04 \pm 0.07 \mathrm{dex}\right)$, which we found was due to an evolving mix of the baryonic components that likely dominate within $r_{2.2}$. While the exact fraction of dynamically mature disk galaxies at a given redshift is debated, the results of Paper I for disks at $z \simeq 1$ contrast markedly with the dispersion-dominated dynamics of massive, star-forming galaxies observed 2-3 Gyr earlier at $z>2$ (Genzel et al. 2006; Förster Schreiber et al. 2009; Jones et al. 2010). In addition to a large increase in scatter, Cresci et al. (2009) show the $z \sim 2.2 M_{*}$-TFR has an offset of $\Delta M_{*} \sim 0.41 \pm 0.11 \mathrm{dex}$ from that found in Paper I. The implication is that star-forming galaxies very rapidly establish their present dynamical state during the intervening period. Thus, resolved dynamical data in the redshift interval $1<z<2$ are needed to make progress, and large samples will be valuable to understand the demographic trends.

There are several observational challenges in pursuing the $M_{*}$-TFR beyond $z \simeq 1$. Multi-object capabilities are essential in creating a large sample to address questions such as those posed above, but these capabilities are only currently available at optical wavelengths. The emission line of choice, [O II $] 3727 \AA$, moves to a wavelength region of low detector sensitivity, and the physical scale being probed is comparable to just a few times the average seeing (a diameter of $5 r_{s}$ at $z \simeq 1.6$ corresponds to $\simeq 2.5 \mathrm{arcsec}$ ). This second paper in our series was motivated by the installation of a deep depletion, red-sensitive CCD on the red arm of the Low Resolution Imaging Spectrograph (LRIS; Oke et al. 1995) which has significantly improved the efficiency in the wavelength interval $8000 \AA-10,000 \AA$ corresponding to [O II] in 
Table 1

Summary of LRIS Observations and Data

\begin{tabular}{|c|c|c|c|c|c|c|c|c|}
\hline Field & $N_{\mathrm{msk}^{\mathrm{a}}}^{\mathrm{a}}$ & $N_{\text {obj }}{ }^{\mathrm{b}}$ & $t_{\exp }^{c}$ & Dates of Obs ${ }^{\mathrm{d}}$ & Seeing & $H S T$ Filters ${ }^{\mathrm{e}}$ & Ground IR Phot ${ }^{\mathrm{f}}$ & IR Catalogg \\
\hline EGS & 2 & $\begin{array}{l}11(3,3) \\
12(2,2)\end{array}$ & $\begin{array}{l}30.0 \mathrm{ks} \\
14.4 \mathrm{ks}\end{array}$ & $\begin{array}{c}2009 \text { Jun } 26-28 \\
,\end{array}$ & $\begin{array}{l}1^{\prime \prime} .05 \\
0.93\end{array}$ & F606W, F814W & Palomar $\left(J K_{s}\right)$ & Bundy et al. (2006) \\
\hline SSA22 & 2 & $\begin{array}{l}13(3,3) \\
12(2,1)\end{array}$ & $\begin{array}{l}22.5 \mathrm{ks} \\
12.0 \mathrm{ks}\end{array}$ & " & $\begin{array}{l}0.78 \\
0.89\end{array}$ & $F 814 W$ & $\mathrm{UH} 2.2 \mathrm{~m}\left(J H K_{s}\right)$ & Capak et al. (2004) \\
\hline GOODS N & 1 & $8(1,3)$ & $34.8 \mathrm{ks}$ & 2010 Apr 5-6 & 0.86 & $\begin{array}{l}F 435 W, F 606 W \\
F 775 W, F 850 L P\end{array}$ & $\begin{array}{l}\text { MOIRCS }\left(K_{s}\right) \\
\text { on Subaru }\end{array}$ & Bundy et al. (2009) \\
\hline GOODS S & 1 & $5(1,1)$ & $25.2 \mathrm{ks}$ & 2011 Feb 28 & $0^{\prime} \cdot 98$ & $\begin{array}{l}F 435 W, F 606 W \\
F 775 W, F 850 L P\end{array}$ & $\begin{array}{l}\text { ISAAC }\left(K_{s}\right) \\
\text { on ESO VLT }\end{array}$ & Retzlaff et al. (2010) \\
\hline
\end{tabular}

Notes.

a Number of masks per field.

b Number per mask and those of which are compact and passive, see Section 3.1.

c Total integrated exposure time per mask.

${ }^{\mathrm{d}}$ Dates observations were taken at Keck I.

e Filters of HST ACS imaging available for size measures.

${ }^{\mathrm{f}}$ Ground-based infrared photometry available for SED fitting, in addition to further optical filters (not shown).

g Reference to photometry matching catalog.

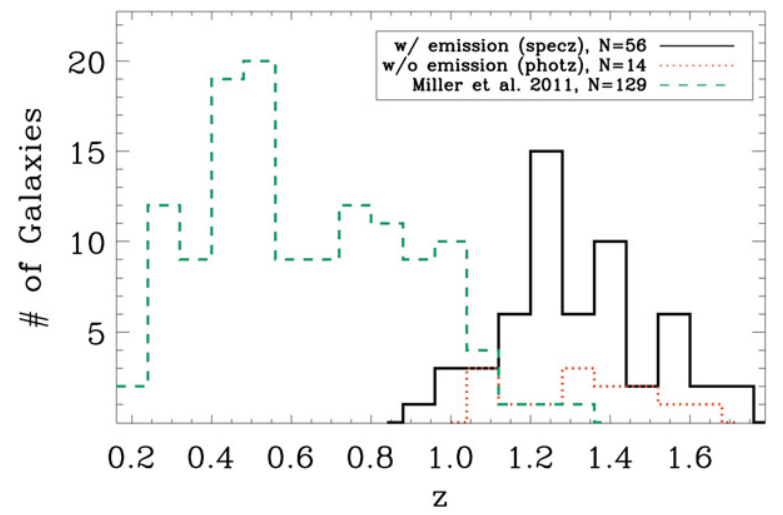

Figure 1. Redshift distribution of 70 galaxies in our new sample compared to that in Paper I. Line emission was detected in most of the $z>1$ galaxies targeted as shown in solid black. The dotted orange line shows the distribution for those galaxies with no detected emission.

(A color version of this figure is available in the online journal.)

the redshift interval $1.0 \lesssim z<1.7$ (Figure 1). This allows us to extend our earlier work to try to understand the transition from the dispersion-dominated systems observed above $z \simeq 2$, and to evaluate the nature of the $M_{*}$-TFR 2 Gyr earlier than that probed in Paper I. We present the results based on a sample of 70 morphologically selected star-forming galaxies for which we have obtained the necessary extended integration times, following the techniques developed in Paper I.

The plan of the paper is as follows: in Section 2, we describe the sample selection criteria, the LRIS data, and the Hubble Space Telescope (HST) Advanced Camera for Surveys (ACS) resolved photometry and stellar mass estimates; Section 3 explains our technique in determining the fiducial rotation velocities, as well as various tests of the chosen method; in Section 4 , we present our results of the TFR from $1.0 \lesssim z<1.7$, and in Section 5 we discuss our results in light of current disk assembly theory and pioneering observations to even higher redshift. Throughout the paper, we adopt a $\Omega_{\Lambda}=0.7, \Omega_{m}=0.3$, and $H_{0}=70 \mathrm{~km} \mathrm{sec}^{-1} \mathrm{Mpc}^{-1}$ cosmology. All magnitudes refer to the $\mathrm{AB}$ system.

\section{DATA}

We selected our sample of spectroscopic targets in a similar manner to that in Paper I so as to span a broad mass and luminosity range. A more detailed look at sample representativeness can be found in the Appendix, but the salient points are summarized in the following section. The necessity of HST ACS imaging for accurate disk size measurements led us to focus on five fields with multi-wavelength coverage for selecting targets: (1) the Great Observatories Origins Deep Survey (GOODS) North and (2) GOODS South fields (Giavalisco et al. 2004), (3) the Small Selected Area 22 (SSA22) field (Lilly et al. 1991; Chapman et al. 2004; Abraham et al. 2007), (4) the Extended Groth Strip (EGS) field (Davis et al. 2005), and (5) the Cosmic Evolution Survey (COSMOS) field (Scoville et al. 2007). A summary of masks, targets, spectroscopic observations, and available space and ground-based imaging in each field can be found in Table 1.

In selecting our targets we applied an apparent magnitude limit of $i_{\mathrm{AB}}<25$ and selected sources in the redshift range $1.0<$ $z<1.7$ (using photometric redshifts when a spectroscopic redshift was unavailable). This led to a sample of 50 targets per LRIS pointing. Potential targets were then visually inspected by at least two of us (R.S.E., K.B., S.H.M.) on the ACS images and potential targets with compact morphology, without diffuse extension, were given low priority but not excluded. ${ }^{5}$ From a prioritized list of typically 25 suitable targets per LRIS pointing, on average $\sim 10$ of these were included per mask due to the requirements of both suitably oriented slits and simultaneous accommodation of unrelated targets for other science campaigns. In designing multi-slit masks for LRIS, we aligned $1^{\prime \prime}$ slitlets to the major axes of our targets as fitted by SExtractor (Bertin \& Arnouts 1996). The position angle (P.A.) of the mask was then selected to minimize slitlet tilts while maximizing target count, and we always ensure that target slitlets are within $\pm 30^{\circ}$ of the mask P.A. We used the 600 liter $\mathrm{mm}^{-1}$ grating blazed at $10,000 \AA$, providing an

\footnotetext{
5 In considering the effect of excluding unresolved or compact sources, it is important to recognize that the dynamical measurements for spatially unresolved galaxies will likely add to the observed scatter through their measurement uncertainties rather than because those sources have an intrinsically larger scatter around the relationship.
} 
instrumental velocity resolution of $58 \mathrm{~km} \mathrm{~s}^{-1}$ at $9000 \AA$ thereby exploiting the sensitive region of the newly installed CCD. The spectroscopic LRIS data were reduced by one of us (A.N.), using techniques described in detail by Newman et al. (2010) with the code developed by Kelson (2003), in which sky modeling and subtraction is carried out with sub-pixel sampling of the background. The seeing, which varied from 0.78 to $1^{\prime \prime} .05$, was measured by taking the weighted average of the FWHM of the alignment stars on each mask.

\subsection{Photometric Components}

With the existing HST ACS data (see Table 1 for availability per field), we conduct our measurements of disk radii and inclination of each galaxy using the reddest available filter $(F 814 W$ or $F 850 L P)$. We ensure that light from the bulge component does not confuse the fitted disk component by conducting a bulge-disk decomposition, where appropriate, similar to our procedure in Paper I.

The HST images were analyzed for photometric components using the GALFIT3 (Peng 2010) least-squares elliptical-fitting method described in detail in Paper I to which the reader is referred. Briefly, we fit an exponential disk component plus a de Vaucouleurs' bulge profile to every galaxy. Those galaxies which yielded unphysical solutions were re-fitted with a single Sérsic profile component, where the Sérsic index ( $n)$ was allowed to vary. Such cases generally represent disk galaxies which are bulgeless and/or more clumpy and irregular than regular well-formed spirals. For the present sample, $\sim 63 \%$ of our galaxies were fitted using a one-component $n$-varying Sérsic profile fit, and $\sim 37 \%$ were adequately fitted with a two-component bulge and disk solution. This mix provides a valuable indication of the morphological distribution of our sample, suggesting less than half are well-formed disks. For comparison, this mix was 60:40 in Paper I.

We ran GALFIT using $H S T$ data of the reddest available filters (noted in Table 1). Where imaging from more than one filter is available, we run GALFIT on each and see $<10 \%$ difference between bands. In our comparisons between the $F 435 \mathrm{~W}$ filter in Paper I and the redder filters, we see no systematic offset, but typically a $<5 \%$ increase in the scatter of the radius fit. We assume an additional uncertainty of this nature exists in the measurements of this data set since we are sampling rest-frame blue-UV light in the majority of the sample. To achieve convergence on the GALFIT parameters and to assess their errors, we run a similar Monte Carlo analysis to that in Paper I. We found the parameter output distributions were much narrower than the input distributions, suggesting convergence. Final parameter uncertainties from the Monte Carlo distributions are better than $10 \%$ on average, and the additional uncertainties are added in quadrature to the observational error and formal fitting errors.

\subsection{Stellar Masses}

Our method of estimating stellar mass in our galaxies follows the work initially presented in Bundy et al. (2005), followed by the analysis presented in Bundy et al. (2009). Further details can be found in those papers.

Briefly, stellar masses are derived using a matched catalog of multi-band ACS, available optical, and ground-based infrared photometry (see Table 1 for ACS and infrared availability per field). A Bayesian code fits the spectral energy distribution (SED) derived from 2"ACS, and ground-based optical and infrared photometry, adopting the best spectroscopic redshift and this SED is compared to a grid of Bruzual \& Charlot (2003) models that span a range of metallicities, star formation histories, ages, and dust content. The stellar mass is calculated from the derived $K$-band mass/light ratio assuming a Chabrier (2003) initial mass function (IMF). The probability for each fit is marginalized over the grid of models giving a stellar mass posterior distribution function, the median of which is the cataloged value. At the magnitudes probed in this survey, the uncertainty inferred from the median $68 \%$ equivalent width of these posterior functions is $0.174 \mathrm{dex}$.

In order to determine the systematic uncertainties arising from combining different sets of photometry into a single sample, we compare mass estimates for our sample in SSA22 using different combinations of the available photometry, e.g., the filters available in EGS. The resulting dispersion in the distribution of stellar mass estimates ranges per galaxy from 0.005 to 0.1 in dex, with a median standard deviation of 0.052 , which we add in quadrature to each galaxy's SED median posterior width as an additional systematic uncertainty. The combined stellar mass uncertainty is better than 0.2 dex in over $80 \%$ of our sample. ${ }^{6}$ As in Paper I we use the stellar mass enclosed within $2.2 r_{s}$, as scaled from the reddest available $H S T$ broadband flux, in order to compare like velocity with like mass. The average reduction in stellar mass at $r_{2.2}$ is $-0.187 \mathrm{dex}$ compared to the total stellar mass estimates to the Kron radius (Kron 1980). For the present sample, we successfully probe down to a minimum total stellar mass of $\log M_{*} / M_{\odot}$ of $8.73 \mathrm{dex}$, with a mean of 10.04 dex.

\section{DYNAMICAL ANALYSIS}

Modeling rotation curves beyond $z \sim 1$ is a challenging endeavor in many respects. In addition to the shift of the diagnostic [O II] $3727 \AA$ emission line into the sky-dominated red spectral region, the more distant galaxies are smaller in angular scale making resolved measurements challenging from the ground without the use of adaptive optics (AO). Prior to the availability of near-infrared (NIR) multi-integral field unit (IFU) instruments (e.g., KMOS on the VLT), multi-slit spectrographs retain several advantages for delivering kinematics for large samples of galaxies over a wide redshift range. Only a fraction of the presently available IFU data sets have been observed with $\mathrm{AO}$, and the typical wider wavelength ranges and multiplexing capabilities of spectrographs such as DEIMOS, LRIS, and the soon-to-be-commissioned MOSFIRE on Keck enable extended exposures on survey sources without a penalty in spectral or spatial resolution. Improved multiplexing of IFUs will mitigate this current deficiency as two-dimensional spectroscopy offers clear advantages in optimal fitting of complex velocity fields (Puech et al. 2008; Jones et al. 2010; Genzel et al. 2011; Förster Schreiber et al. 2011; Contini et al. 2012). For now, multi-slit spectrographs retain a key advantage in survey efficiency. Here, we exploit the improved red sensitivity of a newly installed LRIS deep depletion CCD from Lawrence Berkeley National Laboratories (Rockosi et al. 2010) to secure resolved dynamics for 70 galaxies in the redshift interval $1<z<1.7$ with a lower average stellar mass range than the many extant IFU samples.

We discuss our motivation in Paper I to determine the rotational velocity at a fixed fiducial radius, and why we choose

\footnotetext{
6 This does not include systematic uncertainties regarding our incomplete knowledge of stellar populations in the various types of galaxies present in this sample at these redshifts - uncertainty which affects all present studies of this nature.
} 
this to be $r_{2.2}$, or 2.2 times the scale radius $\left(2.2 \times r_{s}\right)$ of the galaxy, as measured from the broadband HST optical filter. A maximum measured velocity, $V_{\max }$, is highly dependent on the inconsistent distribution of emitting gas in a given galaxy for studies based on nebular emission lines such as ours. So it is important instead to base a measurement for the TFR at a consistent location in the disk galaxy such as $V_{2.2}$, the velocity found at $r_{2.2}$ (further justified in Paper I, and references therein). In the following we will briefly review our methodology for extracting rotation curves and determining the velocity, $V_{2.2}$, at this fiducial radius, as well as testing the uncertainties given the increased distance of our sample compared to the Paper I sample.

\subsection{Rotation Curve Modeling}

As in Paper I, we adopt the empirically motivated arctan function as the starting point of our modeling procedure, viz:

$$
V=V_{0}+\frac{2}{\pi} V_{a} \arctan \left(\frac{r-r_{0}}{r_{t}}\right),
$$

where $V_{0}$ is the central or systematic velocity, $r_{0}$ is the dynamic center, $V_{a}$ is the asymptotic velocity, and $r_{t}$ is the turnover radius, which is the transitional point between the rising and flattening part of the rotation curve (Courteau 1997; Willick 1999).

Paramount to modeling emission lines for rotation curves is that the emission being modeled is adequately resolved. There are two situations in which this may not occur: (1) the angular extent of the galaxy is insufficient compared to the seeing and spatial sampling of the instrument, and (2) where the galaxy may be sufficiently extended in broadband imaging but the spectroscopic emission remains unresolved. Our morphological selection has effectively de-selected the first category but we must now deal with the second.

Ideally, the line emission must extend beyond the seeing disk. To robustly model the dynamics with the arctan function we have generally found that this corresponds to a simple criterion that the line emission must extend to at least one disk scale radius $\left(r_{s}\right)$, as measured by the broadband photometry. By testing our modeling code with simulated data we found in Paper I that too many possible models are consistent with the data if this simple criterion regarding the emission line extent is not met. By progressively removing flux from the outer disk emission in the current LRIS data, we have confirmed this result from Paper I. We thus applied this criterion to define that subset of our sample for which emission line data can be appropriately modeled. Studies which do attempt to dynamically model unresolved kinematics will very likely introduce scatter into their scaling relations. Unresolved rotational velocity gradients, in both IFU and multi-slit data sets, will propagate through the instrument as increased dispersion, degenerate with the intrinsic velocity dispersion in the optical and NIR-emitting gas of the galaxy. In particular, if the unresolved velocity gradient of the inner solid body rotation is the only part of the galaxy with sufficient signal to noise, fitting a model such as the arctan function in Equation (1) will result in poorly constrained extrapolated velocities toward the outer disk, e.g., any velocity wishing to sample the flat part of the rotation curve for a TF study (Courteau 1997; Miller et al. 2011).

Of the 70 spectra discussed earlier, 60\% (42) show emission which is sufficiently extended according to the above criterion from which a rotation curve can be modeled. Of the remainder, $20 \%$ (14) have detectable line emission but it is too compact, i.e., does not extend above $r_{s}$, and $20 \%$ (14) appear to be passive without emission. This 60/20/20 composition of extended, compact, and passive spectral emission, respectively, is similar to the equivalent mix $(55 / 25 / 20)$ seen in Paper I, and as before these three sub-samples are not statistically different in redshift (Figure 2). For the spectrally compact sample, it appears that 6 out of the 14 galaxies are simply too small to resolve, and the other 8 are well extended in their broadband optical disk, but are only undergoing significant star formation in their central regions, within 1 disk scale radius. These spectrally compact objects as well as the spectrally passive objects have larger stellar masses by 0.2 dex than disks with extended emission. Spectrally passive disks have only slightly redder $I-K$ colors (by $>1 \sigma$ ) than the distribution width of extended emission galaxies, but show no significant difference in size compared to those with extended emission.

As in Paper I (see for full detailed modeling procedure), we blur the arctan function in both the spatial and spectral dimensions to account for seeing and instrumental dispersion, respectively; these are highly degenerate in many cases. In order to attempt to break this degeneracy we measure the positiondependent dispersion from the LRIS spectra in each galaxy, and separately measure the seeing from the alignment stars in each slit mask. In the model, we implement the dispersion measured from the spectrum, reduced by a factor fit to remove additional dispersion of the line from blurring by seeing. We then blur the model by the seeing, and re-normalize the flux across each spatial bin (see Figure 3 for examples). The factor to account for the effect of the seeing in the dispersion is fitted by the Levenberg-Marquardt least-squares solver, along with the parameters of the underlying arctan function shown in Equation (1). We then correct the extracted values of $V_{2.2}$ at $r_{2.2}$ for the effects of disk inclination with the minor-to-major axis ratio $b / a$ derived from our GALFIT results.

For 35 galaxies (half our original sample) our spectra are sufficiently deep that we can robustly follow the line emission to $r_{2.2}$ (after accounting for seeing). For the remaining seven galaxies with extended line emission, we are forced to infer $V_{2.2}$ by extrapolating beyond the point of maximum emission extent in the best-fit rotation curve. The error budget for this modeling method is discussed in Paper I, and we discuss further uncertainties regarding this specific application of our spectral models below.

\subsection{Testing the Model}

At a redshift $z \simeq 1.5$, a typical scale radius of $4 \mathrm{kpc}$ is only $\sim 0.5$ arcsec and so clearly seeing and instrumental dispersion blur much of the detail in our LRIS spectra. As it is not always clear visually that there is an intrinsic rotation curve with a characteristic turnover point, we need to demonstrate the reliability and precision of our modeling technique.

To facilitate this, we selected galaxies in the redshift range $0.85<z<1.3$ studied with DEIMOS in Paper I and resampled these data to the spatial and spectral resolution of the LRIS data. With the cosmology assumed, the average angular scale of the LRIS galaxies (at $\langle z\rangle=1.31$ ) is $8 \%$ smaller than that for our chosen DEIMOS sample (at $\langle z\rangle=0.98$ ). To account for differences between Paper I and the present survey, we therefore resample for the different spatial pixel scales (0'.1185 with DEIMOS to 0.27 with LRIS) and match the LRIS spectral dispersions $\left(58 \mathrm{~km} \mathrm{~s}^{-1}\right.$ at $9000 \AA$ with $27.9 \mathrm{~km} \mathrm{~s}^{-1}$ for each pixel). We then analyzed the resampled DEIMOS data using the same rotation curve fitting procedure as we use for the LRIS analysis, and the results can be seen in Figures 4 and 5. 


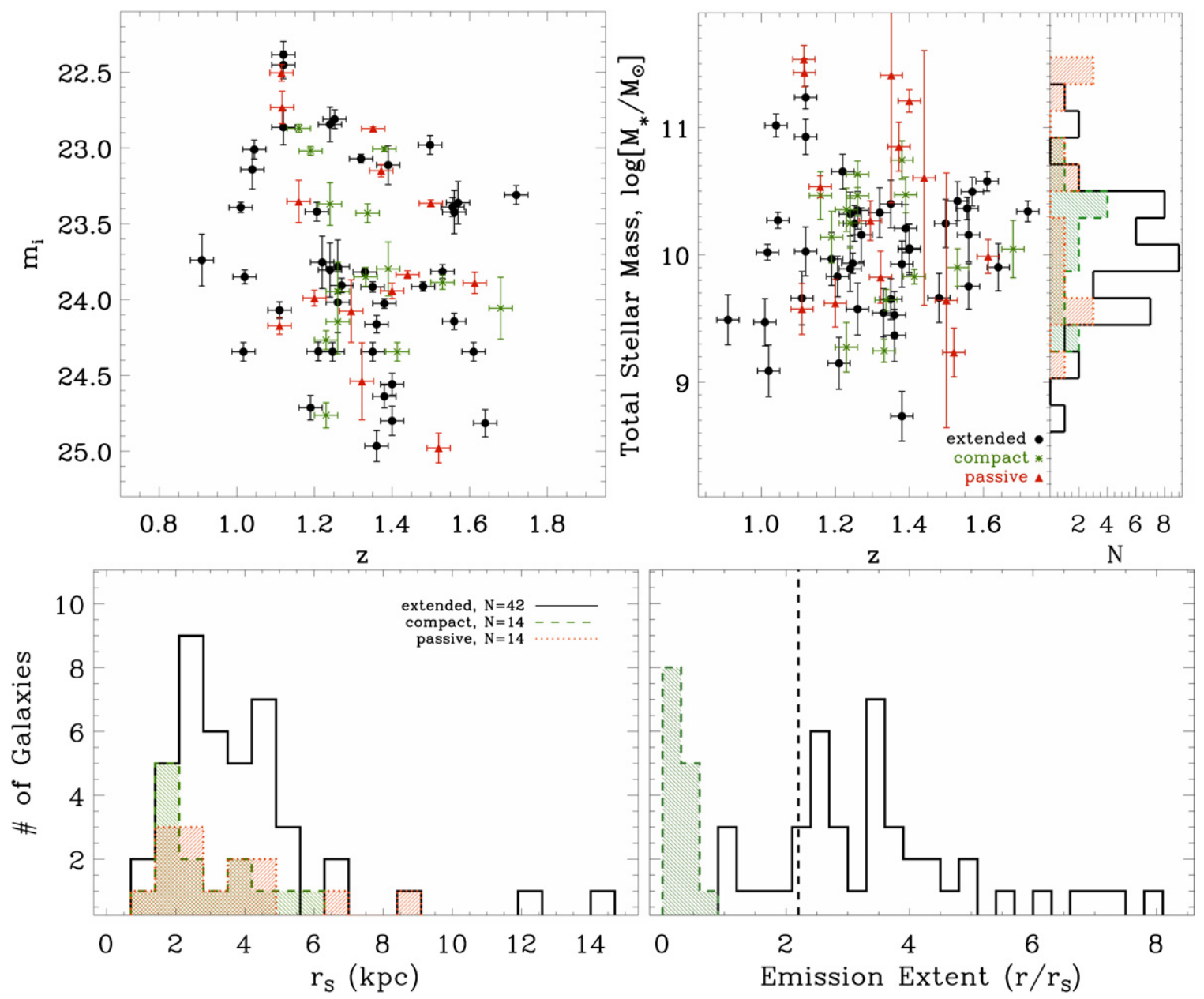

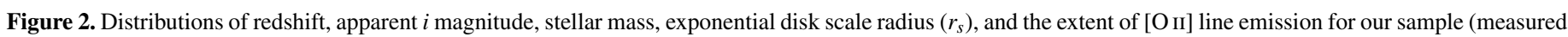

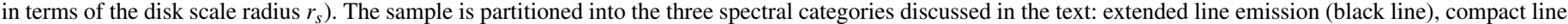

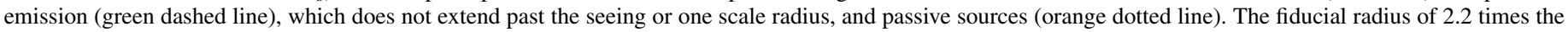
disk scale radius $r_{s}$ is plotted with the vertical black dashed line. See Section 3 for further details.

(A color version of this figure is available in the online journal.)

Encouragingly, when we subtract the recovered $V_{2.2}$ of the resampled DEIMOS data from that of the original data, we find a weighted mean of less than $0.002 \pm 0.009 \mathrm{dex}$, and the scatter in the relation between the two is $0.098 \mathrm{dex}$, which is similar to the rms scatter found in the TFR established locally and in Paper I. Resampling objects at $z \sim 1.0$ to the angular diameter distance of that found at $z \sim 1.3$ did not make significant changes in the recovered $V_{2.2}$, given this corresponds to only a $8 \%$ reduction in the angular scale.

We also tested the data with models simpler than the arctanbased model. This addresses a possible concern that we might be overfitting the data given the apparent lack of resolved detail at this somewhat higher redshift. By assuming a linear fit to the emission at LRIS resolution, the scatter in the resulting estimated $V_{2.2}$ was as large as $1 \mathrm{dex}$ in $\log \left[V / \mathrm{km} \mathrm{s}^{-1}\right]$ compared to that of the arctan-based fits at DEIMOS resolution. We also tested whether we could recover $V_{2.2}$ by measuring $d V / d r$ at intervals of 0.1 scale radii along the disks, but the scatter in the estimated $V_{2.2}$ was on average $0.5 \mathrm{dex}$, depending on the radial extent of the emission.

\section{RESULTS}

The primary result of this paper, the $M_{*}$-TFR for the redshift interval $1<z<1.7$, is shown in Figure 6. As in Paper I, we plot the enclosed stellar mass within $r_{2.2}$ against the deprojected rotational velocity $V_{2.2}$. To interpret the relationship with respect to our earlier work, we fit a linear regression using a least-squares method incorporating the intrinsic scatter $\left(\sigma_{\text {int }}\right)$ added in quadrature to that of the velocity and accounting for errors in both the ordinate and abscissa. As in Paper I, we focus our analysis on the inverse fit, assuming velocity as a function of mass. However, following traditional convention, the relation is displayed with $V_{2.2}$ on the $x$-axis and $M_{*}$ on the $y$-axis.

Our linear fit can be written as

$$
\log \left(\frac{M_{*}}{M_{\odot}}\right)=a+b \log \left(\frac{V_{2.2}}{\mathrm{~km} \mathrm{~s}^{-1}}\right)-\log \left(\frac{M_{0}}{M_{\odot}}\right),
$$

where $M_{0}=10^{10} M_{\odot}$. With a slope $b=3.869$ fixed from that derived for the total sample in Paper I, we find that $a=1.692 \pm$ $0.060, \sigma_{\text {int }}=0.080 \mathrm{dex}$ in $\log \left[V / \mathrm{km} \mathrm{s}^{-1}\right]$, and the total $\mathrm{rms}$ is 0.117 dex, which is reasonable considering the median error is 0.102 dex. In stellar mass, this corresponds to $\sigma_{\text {int }}=0.310 \mathrm{dex}$ in $\log \left[M_{*} / M_{\odot}\right]$ and an rms of $0.452 \mathrm{dex}$ (where the median error is $0.186 \mathrm{dex}$ ).

While we recover a zero point for the fixed-slope $M_{*}$-TFR similar to that found in Paper I for $0.2<z<1.3$, the scatter in the relation has apparently increased as much as $60 \%$ as compared to the local relation. Part of this increased scatter could 


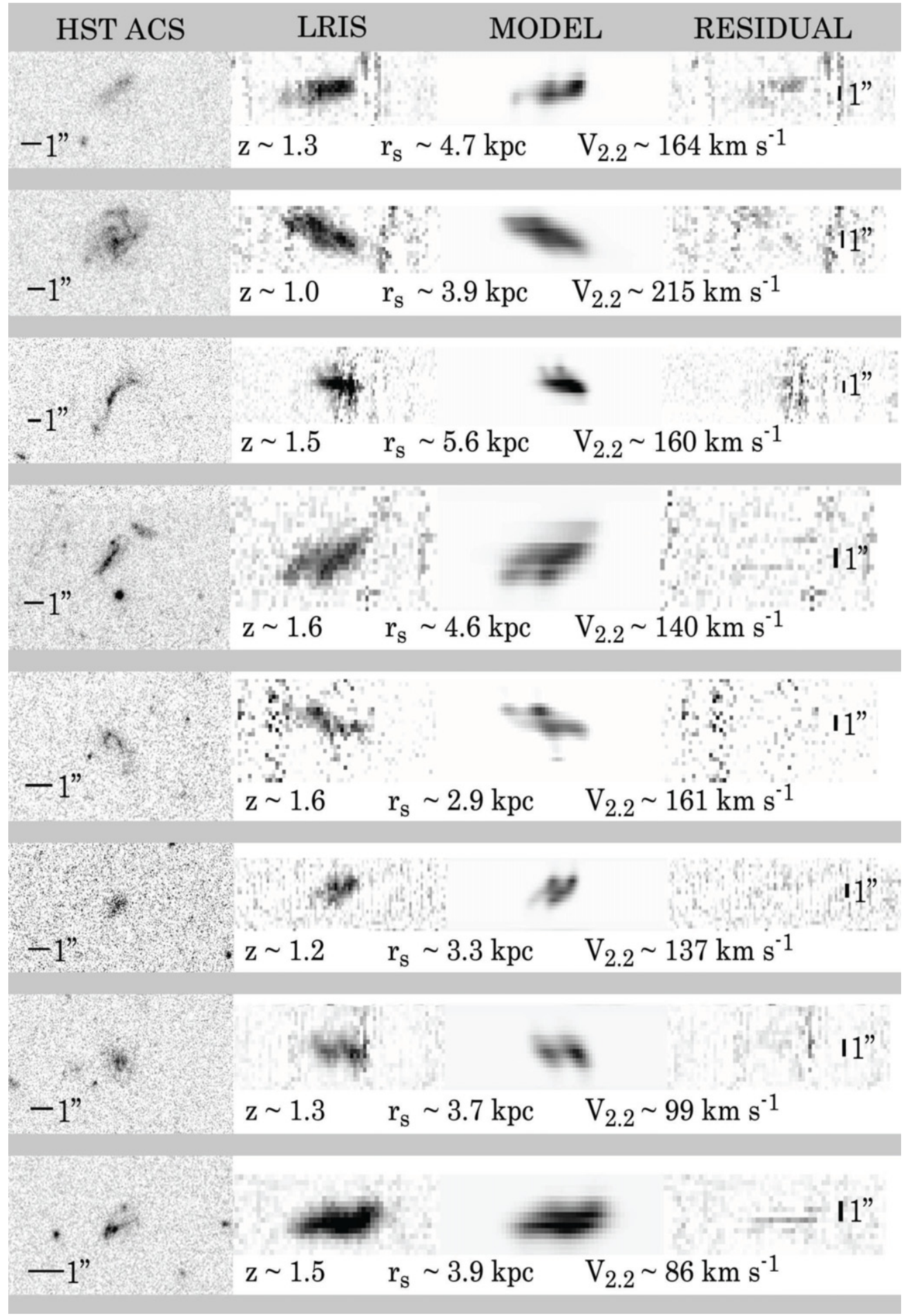

Figure 3. Examples of our kinematic analysis: (from left to right) the HST image of the galaxy in ACS F850LP or $F 814 W$ filter; the two-dimensional [O II] data in the reduced LRIS spectra; the modeled emission trace using an arctan function blurred by both the measured seeing and dispersion (Section 3.1); the residual obtained by comparing the latter two signals.

be attributed to the more challenging observations undertaken with LRIS. We note, for example, that the additional scatter introduced by using LRIS rather than DEIMOS as described earlier $\left(\sim 0.098 \mathrm{dex}\right.$ in $\left.\log \left[V / \mathrm{km} \mathrm{s}^{-1}\right]\right)$ implies our intrinsic scatter could be as low as 0.063 dex. Given the uncertainty in the scatter is about 0.01 dex, this value is not significantly different than that found in Paper I (0.058 dex; Miller et al. 2011). So while it is possible that the relation is less well defined by a broadening of up to $60 \%$ compared to $z \sim 0$, where average scatter is found to be $\sim 0.05$ dex (i.e., Pizagno et al. 2005; Reyes et al. 2011), we cannot rule out the possibility that the tightness of the relation in this study is the same as that found locally.

We also show in Figure 6 the stellar masses for two important subsets of the originally targeted sample whose resolved dynamics cannot be determined. The first category are the 14 spectrally passive objects which are clearly some of the most massive galaxies in the sample. Conceivably these passive galaxies are evolving off the TFR and transitioning onto the red sequence. 


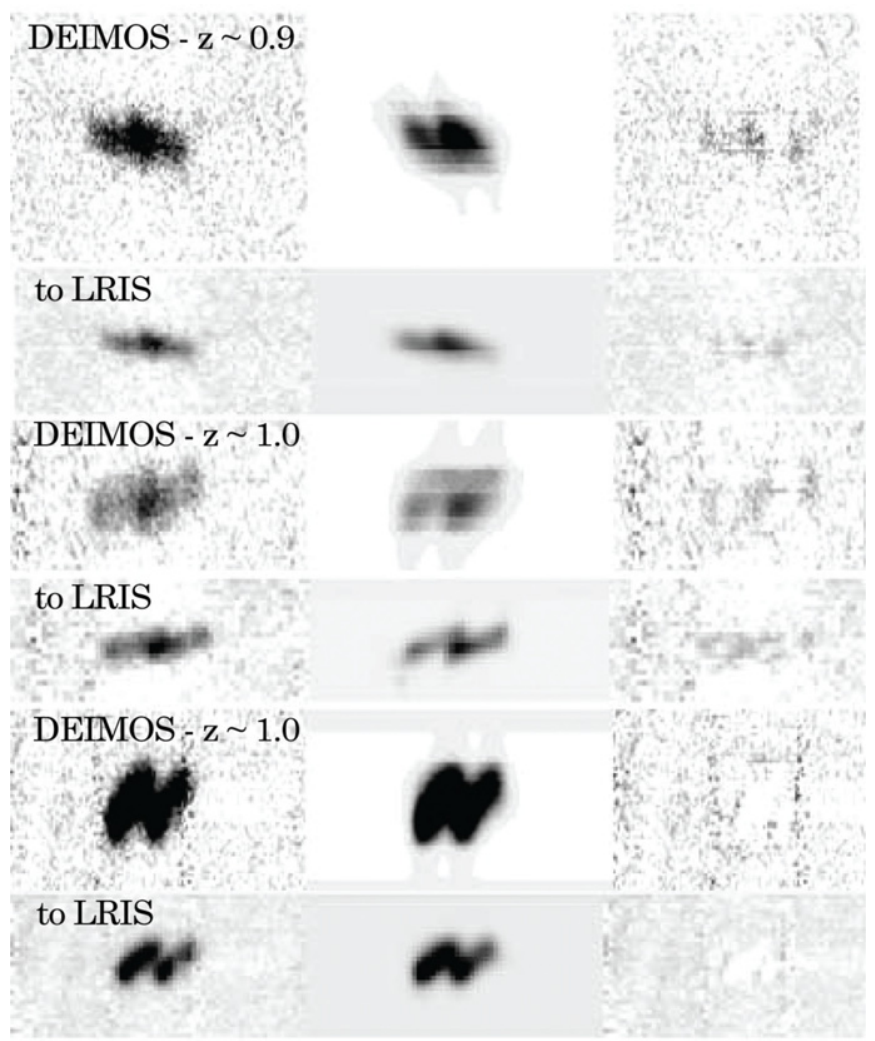

Figure 4. Three galaxies at $z \sim 1$ observed with DEIMOS, compared to a simulated observation at the different spatial and spectral resolution of the LRIS instrument directly below. From left to right, the columns show (i) the spectral data, (ii) the best-fit model, and (iii) the residual of the data from the model.

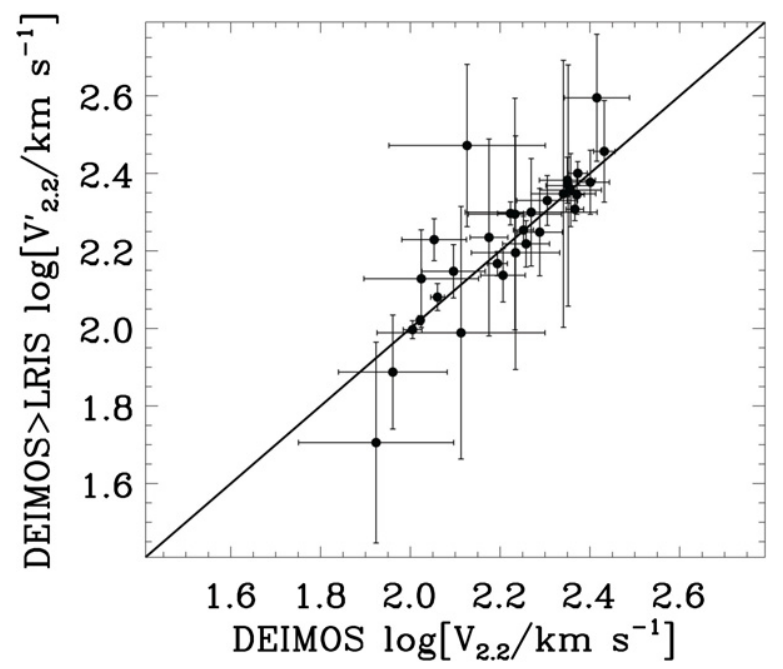

Figure 5. Testing the method: for a subset of galaxies from Paper I in the redshift interval $0.85<z<1.3$ we resample their original DEIMOS spectra to the spatial and instrument resolution of LRIS and compare the derived $V_{2.2}$ with the original measures (see the text for details).

On the other hand, the stellar masses of the spectrally compact objects do not significantly differ from that for main sample displaying suitably extended emission. Most of these galaxies appear to have extended disks in the HST ACS images, although star formation appears to have largely shut off in their outer disks as evidenced by their spectrally compact nature. Conceivably, these galaxies could comprise systems with actively forming bulges, up to a few times $10^{10} M_{\odot}$ in stellar mass.

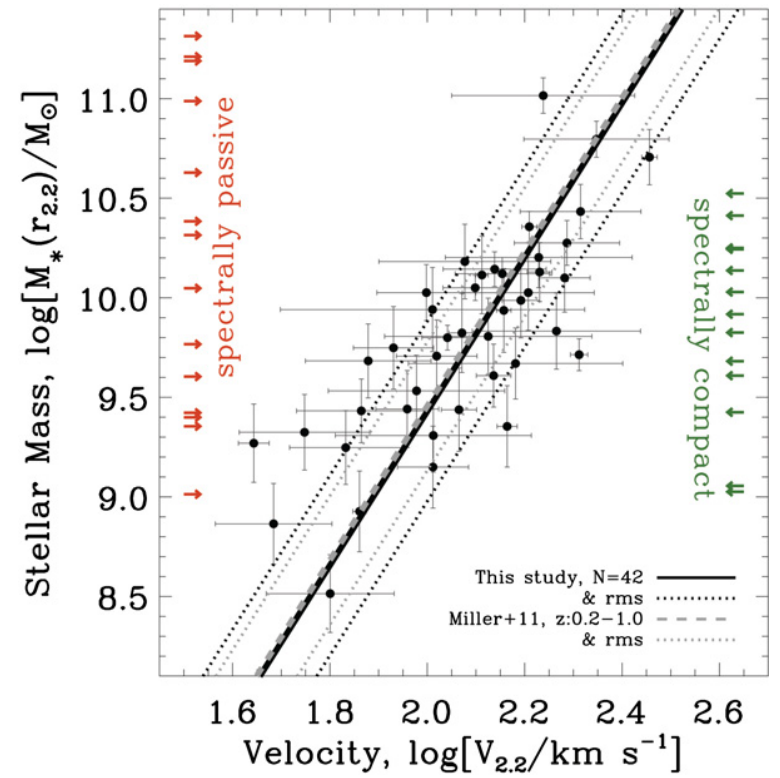

Figure 6. Stellar mass Tully-Fisher relation ( $M_{*}$-TFR) derived for 42 suitable galaxies in the redshift interval $1<z<1.7$. The least-squares inverse fit relation is shown as a solid black line and the rms scatter is displayed as a pair of dotted lines. The best-fit $M_{*}$-TFR found from $z \sim 0.2$ to $z \sim 1$ in Paper I is shown as the dashed gray line (which is almost coincident with the results of this study). The rms scatter around the Paper I fit is shown by the pair of gray dotted lines. Although the zero point has not changed in the intervening $2 \mathrm{Gyr}$, the apparent scatter has increased. We also show with arrows the stellar masses of two categories of galaxies for which we cannot extract measurements of $V_{2.2}$ (see Section 3.1 for details).

(A color version of this figure is available in the online journal.)

Finally, to place our $M_{*}$-TFR in the context of earlier work, Figure 7 summarizes recent results for the $M_{*}$-TFR over the full redshift range $0<z<3$. Notwithstanding possible selection biases discussed in Section 5, it is illustrative to consider this figure. Evolution in the $M_{*}$-TFR zero point can be normalized with respect to the local study of Reyes et al. (2011), calibrated from the parent sample which came from the Sloan Digital Sky Survey (SDSS), and who also adopt the same $V_{2.2}$ parameterization used in this series. To ensure an adequate comparison, we re-fit our data using the Reyes et al. (2011) slope (which we note is not significantly different from the slope we find). Because Reyes et al. (2011) use the total mass rather than that enclosed at $r_{2.2}$, we move their zero point by $-0.187 \mathrm{dex}$ in stellar mass to account for the difference in the enclosed mass as compared to the total stellar mass. We make a further correction to account for the different IMF assumed, since Reyes et al. (2011) adopted a Kroupa (2002) IMF, which both sets of authors agree accounts for a 0.05 dex decrease compared to our assumed Chabrier (2003) IMF. In total, this is a $-0.137 \mathrm{dex}$ calibration between the Reyes et al. (2011) study and our work, resulting in only a marginal change in the $M_{*}$-TFR zero point since $z \simeq 1.7$. Formally, the linear regression shown in Figure 7 yields a stellar mass zero-point change of only $0.02 \pm 0.02 \mathrm{dex}$ over the last 9.8 billion years.

We also include results at $z \sim 2.2$ and $z \sim 3$ from Cresci et al. (2009) and Gnerucci et al. (2011), respectively, which suggest a surprisingly rapid and significant evolution beyond $z \sim 1.7$. However, the average stellar mass in the Cresci et al. (2009) sample is $6.3 \times 10^{10} M_{\odot}$ and ranges from 0.62 to $31.6 \times 10^{10} M_{\odot}$, whereas our sample average is $1.3 \times 10^{10} M_{\odot}$ and ranges from 0.05 to $17.2 \times 10^{10} M_{\odot}$ (both using a Chabrier IMF). 

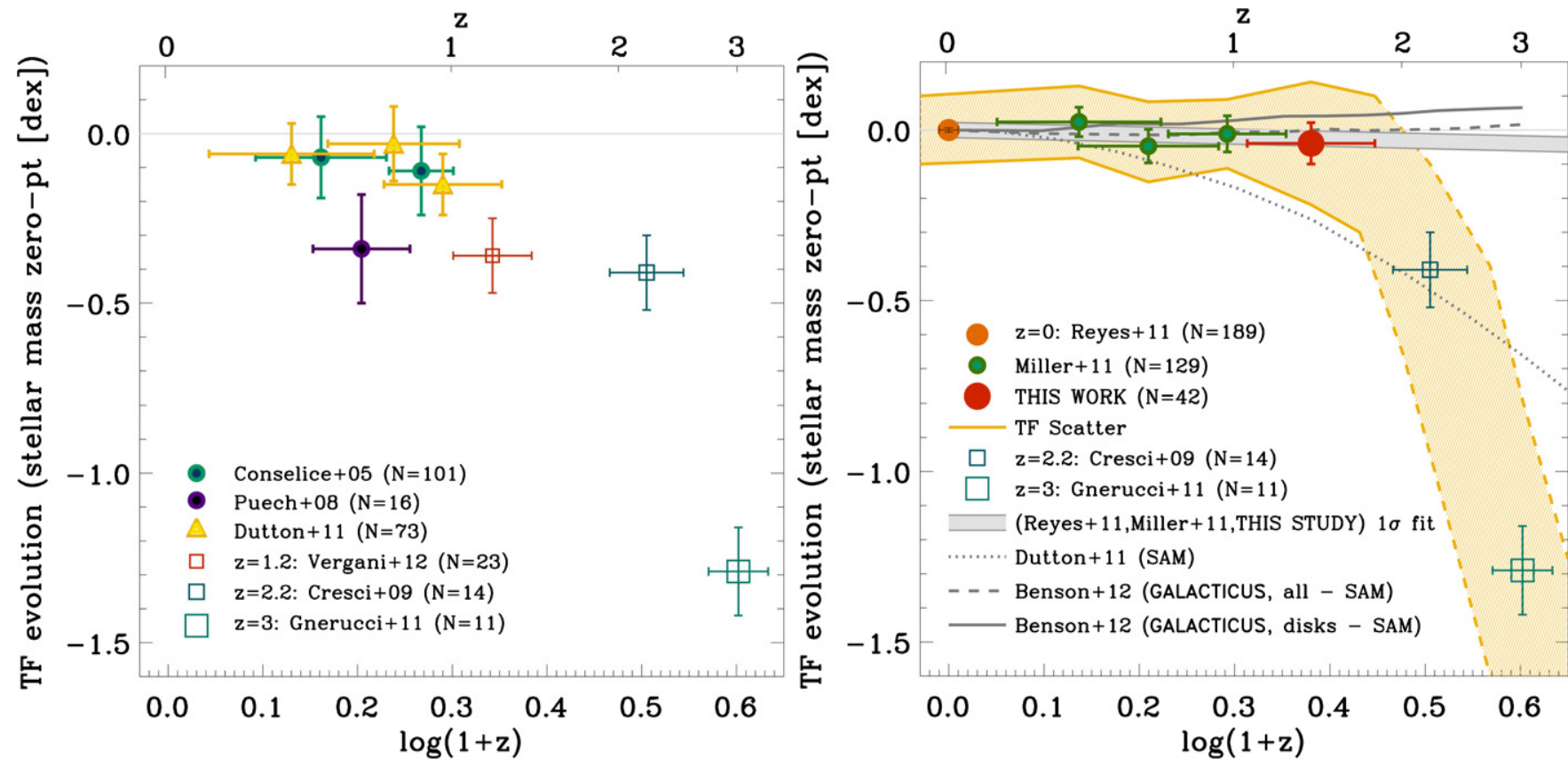

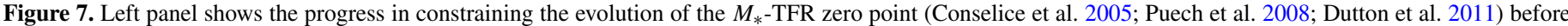

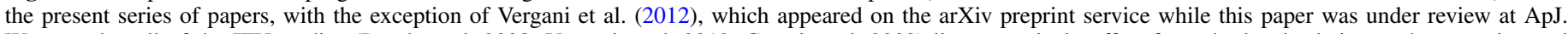

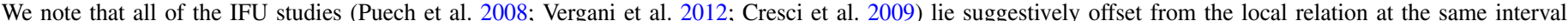

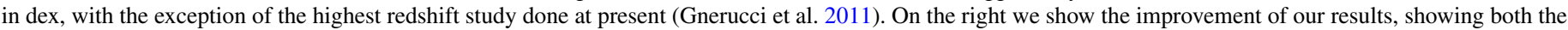

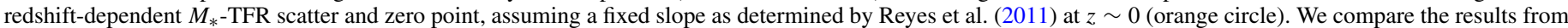

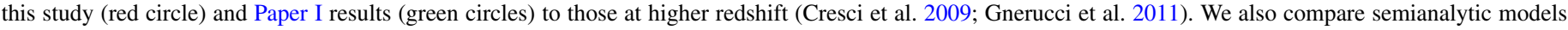
(SAM) from Dutton et al. (2011) and Benson (2012; GAlacticus). For a full discussion of this plot, please see Sections 4 and 5.

As a theoretical comparison we present preliminary $M_{*}$-TFR evolution results computed using GaLACTICUs v0.9.1 r631 ${ }^{7}$ (Benson 2012), which includes various modules for star formation and active galactic nucleus feedback as well as state-ofthe-art theoretical predictions for baryon-halo evolution. Shown in Figure 7 are GALACTICUs models for both disk-dominated galaxies (bulge-to-total mass fraction $<0.3$ ) and all galaxies. In each case, the mean zero points correspond to galaxies with stellar masses between 0.5 and $2 \times 10^{10} M_{\odot}$. Interestingly, the results from models of all galaxies shows a similar, non-evolving trend in the $M_{*}$-TFR zero point, whereas the disk-only subsample of the models departs in evolution to the slow side of the observed relation as redshift increases, but never more than 0.1 dex-even to $z \sim 3$. In this figure we also include the semianalytical model of Dutton et al. (2011), which is consistent with a sample subset of the results originally presented in Kassin et al. (2007; $N=73$ from 544 galaxies). Their projected evolution departs from the results at $z \sim 1$ in this study and Paper I.

\section{DISCUSSION}

The major goal of this paper was to extend our earlier work in Paper I to higher redshifts to determine the rate at which disk galaxies arrive on the TFR. Figure 7 presents a puzzling discontinuity in behavior over a relatively narrow period of cosmic history. A key uncertainty in the interpretation of this figure, however, is that not all of the sample satisfies our criterion of providing a reliable estimate of $V_{2.2}$. Although we have maintained continuity in our selection techniques from Paper I to this paper in the redshift interval $z \simeq 1.0-1.7$, similar criteria

\footnotetext{
7 Computed with an input parameter file which is available from http://sites.google.com/site/galacticusmodel/downloads/parameter-sets
}

of morphological appearance or extended emission lines are difficult to usefully apply to the star-forming galaxies being studied beyond $z \simeq 2$. As redshift increases in Figure 7, some subset of a mass-selected or a star formation rate (SFR) limited population is likely not included in the TF analysis.

Such a selection bias may be more or less important depending on the physical model we wish to test. If disks grew from chaotic beginnings, where clumps collide at the centers of dark halos (van den Bergh et al. 1996; Abraham et al. 1997; Elmegreen \& Elmegreen 2005), then the TFR will continue to evolve as the chaotic behavior and mergers (Hammer et al. 2005) transition into more regular accretion onto well-formed disks. In this scenario, we could interpret the lack of observed evolution as the result of a bias toward "mature" systems at all redshifts. However, since we expect high SFRs in this violent phase, these less mature systems are more likely to be included in our analysis, unless dust obscuration or possible nuclear concentration of star formation makes extended emission lines difficult to detect (i.e., our spectrally compact and passive targets).

Alternatively if disks form predominantly via smooth accretion over time, they could easily evolve along self-similar dynamical relations, making selection effects much less important in understanding the lack of evolution. How then could we interpret the more drastic offsets implied by Figure 7? Even with smooth, cold accretion (e.g., Dekel \& Birnboim 2006), if galaxies formed stars more vigorously and erratically from $z \sim 2$ to 4 than at present times (e.g., Förster Schreiber et al. 2006; Genzel et al. 2008), then a tight $M_{*}$-TFR would be delayed until disk properties are homogenized across the observed populations-similarly for a transition from dynamically hot objects to disks which are primarily rotationally supported. Any inhomogeneity, including differences or changes in: 
1. rotational versus dispersion support;

2. baryonic mass-to-light ratio across the disk;

3. baryonic versus dark matter fraction;

4. specific SFR;

5. gas fraction or gas reservoir size and distribution;

across redshift bins should lead to an evolution in the $M_{*}$-TFR. While various combinations of the above characteristics are likely correlated, they may not evolve in tight lock step. Thus, the emergence of a recognizable $M_{*}$-TFR at $z \simeq 1.5$ is already a result of significance-even if it only applies to some subset of the star-forming population.

At $z \sim 3$, Gnerucci et al. (2011) argue that the nascent TFR has a scatter over an order of magnitude larger than what we observe at $z \simeq 1$. Given that the angular momentum properties are shown to be similar to those at $z<1$ (Bouché et al. 2007), if the sources targeted by Cresci et al. (2009) were less efficient star formers than those studied here, perhaps this could explain the marked stellar mass deficiency indicated in Figure 7. However, the earlier systems display higher gas fractions and SFRs (Tacconi et al. 2010), and considering that they may have additional dynamical mass supported by higher velocity dispersions only makes this discrepancy worse. Studies of less massive sources at $z \sim 2$ and beyond as well as better constraints on the physical sizes of these components will be valuable in resolving this possible discrepancy.

Cresci et al. (2009) and Gnerucci et al. (2011) note the decreased rotation-to-dispersion velocity fraction $(V / \sigma)$ support in galaxies at $z \sim 2$, as shown also in Förster Schreiber et al. (2009), as key in explaining the observed $M_{*}$-TFR evolution. However this picture, which posits a greater degree of turbulent support in thicker disks at high redshift, should result in less rotational motion per unit stellar mass at earlier times, in contrast to the trend shown in Figure 7. Given the negative $M_{*}$ zero-point evolution observed, a reduced $V / \sigma$ at higher redshift would thus imply a substantial evolution in the gas fraction. Simultaneous changes in the dark matter fractions, baryonic mass-to-light ratios, and disk geometries may lead to a less severe evolution in gas fraction. Understanding how these characteristics could conspire to keep the $M_{*}$-TFR from more dramatic changes can be addressed by more sophisticated tools.

Comparing the relative evolution in our study to that of semianalytical models can thus provide interesting interpretations as to the predicted interplay between baryons and dark matter over these epochs. We note that the model of Dutton et al. (2011) departs significantly from the observed trends before $z \sim 1$, whereas the Benson (2012) GALACTICUs models with several more forms of baryonic feedback are a closer match. This suggests that understanding the subtleties in the baryonic physics of galaxies is vitally important to our understanding of galaxy assembly via tools like the TFR. In fact, Dutton et al. (2011) see much less evolution in their baryonic TFR over the same redshift range, signaling the effect of evolving gas fractions in conjunction with their stellar components. Since the evolution of the dark halo drives the self-similar evolution in disk circular velocity in their model, the evolution present in both their baryonic and $M_{*}$-TFRs is being driven by dark matter, similar to the results of Somerville et al. (2008). However, we find in Miller et al. (2011) that baryons may be the dominant driver of dynamics within $r_{2.2}$ and, furthermore, the dominance of dark matter would stabilize the disk against the intense star formation which is observed (Genzel et al. 2008). Because the role of gas in disk galaxies throughout their formation is not adequately understood across a wide mass range at high redshift, it is likely that further work and exploration of the evolution of the baryonic TFR will help to explain why we see so little evolution in the $M_{*}$-TFR since $z \sim 1.7$, especially since we suppose that gas fractions evolve with time (Tacconi et al. 2010), as well as the dark halo response to baryonic matter.

Our main findings can be summarized as follows.

1. Using LRIS on Keck I and techniques discussed in Paper I, we determine reliable rotational velocities using [O II] emission for 42 of 70 targeted star-forming galaxies selected from the redshift range $1.0 \lesssim z<1.7$. We test the reliability of our extracted velocities with reference to our data at higher resolution and lower redshift.

2. We find that the stellar mass TFR for this sub-sample is well determined with up to $60 \%$ increase in scatter compared to the local relation defined by Pizagno et al. (2005). Considering also the relations found in Paper I and locally, we find little zero-point shift corresponding to $\Delta M_{*}=0.02 \pm 0.02$ dex from $z=1.7$ to $z=0$.

3. Concerning the 28 galaxies which were not included in the above analysis, 6 are unresolved both in their broadband imaging and in their spectra, and 14 are spectrally passive. Although the remaining eight show extended, resolved broadband imaging, the emission is insufficiently extended to resolve by LRIS with the extended exposure times of this study. We consider the impact of excluding these sources on our derived TFR and note their properties.

4. The modest evolution seen in the TFR over $0<z<1.7$ contrasts markedly with results emerging at $z>2$. At face value it seems there is a dramatic change in the kinematic properties over a very short period of cosmic history; however a wider range of sample stellar masses is required at $z>2$ to understand the validity and significance of this result.

While this paper was under review for publication, a study appeared on the arXiv.org (astro-ph) preprint service, accepted for publication (Vergani et al. 2012), which plots 23 galaxies from the MASSIV survey onto a stellar mass TFR with similar or larger scatter as that found in our relation $\left(\sigma_{\text {int }}=0.32\right.$ or 0.52 and $\mathrm{rms}=0.48$ or 0.72 ) and little to some evolution in the stellar mass zero point $(-0.05 \pm 0.16$ dex or $-0.36 \pm 0.11)$, depending on the Bell \& de Jong (2001) or Pizagno et al. (2007) slope adopted, respectively. While the kinematics data were obtained on the SINFONI IFU, only five of these objects were observed using AO (and only two in the sub-sample used to plot the stellar mass TFR). In their TF constraints cited above, they exclude over half of their sample where significant emission was detected, not only when the emission is unresolved, as in our study, but also when their velocity field is not well described by a symmetrically rotating disk, the kinematic P.A. differs significantly from the morphologically derived major axis, or when their measured $v / \sigma<1$ (all criteria which are not included in our study). The addition of the modeled velocities in these excluded galaxies adds considerably to the apparent scatter in the Vergani et al. (2012) stellar mass TFR, whereas our inclusion of merger-like systems or galaxies where rotation curve centers appear offset to the HST-derived centers do not appear to result in the same large scatter. This may be due to their use of $V_{\max }$, described in this work, Paper I, and e.g., Courteau (1997) as potentially leading to the underestimation of the true intrinsic maximal rotation velocity because of its dependency on the distribution of emitting gas in the disk, inconsistent between galaxies in a diverse sample such as theirs. Also we find that assuming 
an intrinsic emission distribution based on broadband optical or NIR HST imaging can often lead to systematic offsets in $\chi^{2}$ minimization dynamical modeling, amplified by the scale of seeing and dispersion in the spectral data, as discussed in Paper I of this series. If this effect is significant for slit-based modeling, then it would be just as damaging if not more so in two-dimensional IFU-based modeling.

If the increased scatter we find in the TFR in this study from $1<z<1.7$ is truly intrinsic, then we should be able to determine what is different in those systems which have not yet arrived on the TFR. In future work, we will more closely examine the various characteristics of disk galaxies in our samples from $0.2<z<1.7$ : their estimated mass components, their dynamics, and their bulge-to-disk morphology. The full release of the WFC3 data in the CANDELS fields (Koekemoer et al. 2011) will allow us to compare our current bulge-to-disk decompositions to those in rest-frame optical at our highest redshifts observed. For example, it has been postulated (i.e., Bournaud et al. 2007; Jones et al. 2010) that the development of a central bulge through inspiraling, unstable clumps is key to stabilizing the disk. Alternatively, morphology could be driven by halo accretion type (hot versus cold), in which the circular velocity shows little dependency on morphology in the recent work by Sales et al. (2011). By examining the $M_{*}$-TFR as a function of the bulge-to-disk ratio and morphology, such hypotheses can be directly tested. Furthermore, an in-depth comparison between semianalytic and hydrodynamic models could provide valuable insight into the observed trends. In doing so, we aim to determine what is driving the emergence of the TFR we observe by $z \sim 1.7$ and, potentially, what in the formation processes of $L^{*}$ disk galaxies is leading them to largely stabilize by $z \sim 1$.

S.H.M. thanks the Rhodes Trust, the British Federation of Women Graduates, the sub-department of Astrophysics and New College at the University of Oxford, and the California Institute of Technology for supporting her work. We thank P. Capak for generously allowing us to use his SSA22 photometry catalog. We also thank A. Benson for preliminary results from the Galacticus model to compare to our observations, and we thank C. Peng for supplying us with GALFIT 3.0. We also thank C. Conroy, A. Dutton, L. Hernquist, J. Gunn, and A. Loeb for helpful discussions on this work. We thank the anonymous referee for improving the quality of this work. The spectroscopic data were secured with the W. M. Keck Observatory on Mauna Kea. We thank the observatory staff for their dedication and support. The authors recognize and acknowledge the cultural role and reverence that the summit of Mauna Kea has always had with the indigenous Hawaiian community, and we are most fortunate to have the opportunity to conduct observations from this mountain.

Facilities: Keck:I (LRIS), HST (ACS)

\section{APPENDIX}

\section{FURTHER SAMPLE DETAILS}

Here we more carefully detail the content of our TF sample, suggesting that we have a representative sample of rotationally supported galaxies at $1<z<1.7$, including irregular and peculiar/interacting galaxies.

For our initial selection based on visual morphology, the reason for excluding unresolved, compact sources is that their dynamical measurements from emission lines are similarly unresolved (and in fact often non-existent). This accounts for an effective removal of about $1 / 5$ of objects, and an additional deweighting of $1 / 10$ of objects which meet the magnitude cut. As seen in the morphological montage in Figure 8, the TF sample includes four objects (1/10 of sample) that would be morphologically classified as early types or nearly unresolved (compact), as well as at least eight objects (1/5 of sample) which would be considered peculiar, interacting, or undergoing a minor merger. This fraction is consistent with the evolving merger rate fraction at this redshift (Cassata et al. 2005; Conselice et al. 2008; Bridge et al. 2010), whether or not the initial visual morphological selection was biased toward mergers.

A different picture is presented in works by Flores et al. (2006), Kassin et al. (2007), and Puech et al. (2008) which cite or suggest higher interacting and merging fractions (at least $45 \%$ of their samples with complex kinematics, with an additional $28 \%$ considered to be perturbed rotators in the latter work), which is used to explain increased scatter in the TFR in these works. While it is well known in local studies that peculiar and interacting galaxies add some scatter to the idealized TFR of a homogenous population (e.g., Tutui \& Sofue 1997; Kannappan et al. 2002; Mendes de Oliveira et al. 2003), this level of scatter is similar to what we find in our work. Rather Flores et al. (2006), Kassin et al. (2007), and Puech et al. (2008) argue for the important contribution of peculiar and interacting galaxies on the larger scatter in their relations. Although methods for identifying mergers vary in more complete samples at the same redshifts, e.g., Kartaltepe et al. (2007) and Jogee et al. (2009) (it is not trivial to compare these due to different timescales of merger signatures; Conselice 2009), we nevertheless believe that measurement uncertainty from decreased signal to noise and resolution contributes to increased scatter in previous intermediate- $z$ studies, rather than an increased scatter arising primarily from disturbed or complex kinematics (see also, e.g., Kannappan \& Barton 2004; Miller et al. 2011). We have attempted in this work and in Miller et al. (2011) to clarify this important difference with extended integrations on spectra and a well-tested dynamical modeling method for deriving intrinsic rotation velocities.

The three objects of overlap between our Paper I sample and that of Flores et al. (2006) and Puech et al. (2008) are all in the most kinematically complex classification of the Flores et al. (2006) scheme, so this suggests that we are not removing objects which may contribute maximally to the scatter seen in the TFR, according to Puech et al. (2008). Instead, as mentioned before, we see a similar amount of scatter as local studies which are inclusive to irregular, perturbed, and peculiar galaxies, i.e., Reyes et al. (2011), which has a sample constructed to be representative based on SDSS.

We find in Paper I for $0.2<z<1.3$ galaxies that with only $1 \mathrm{hr}$ of data, $1 / 6$ of the sample would have been in our spectrally compact category and $1 / 3$ of the sample would have been considered passive in emission, i.e., not detected at all by DEIMOS. So over half of the sample that we include in our TFRs would be prematurely excluded in samples that make a significant emission cut based on $\sim 1 \mathrm{hr}$ redshift surveys from similarly sensitive instruments/telescopes as DEIMOS/Keck, such as the $[\mathrm{O}$ II] emission criteria used to construct the MASSIV survey sample (Contini et al. 2012; Vergani et al. 2012).

While in Paper I we implement a slitlet P.A. offset (from major axis) velocity correction, significant in only $10(8 \%)$ of objects, no such corrections were found necessary in this study (see Figure 8). 


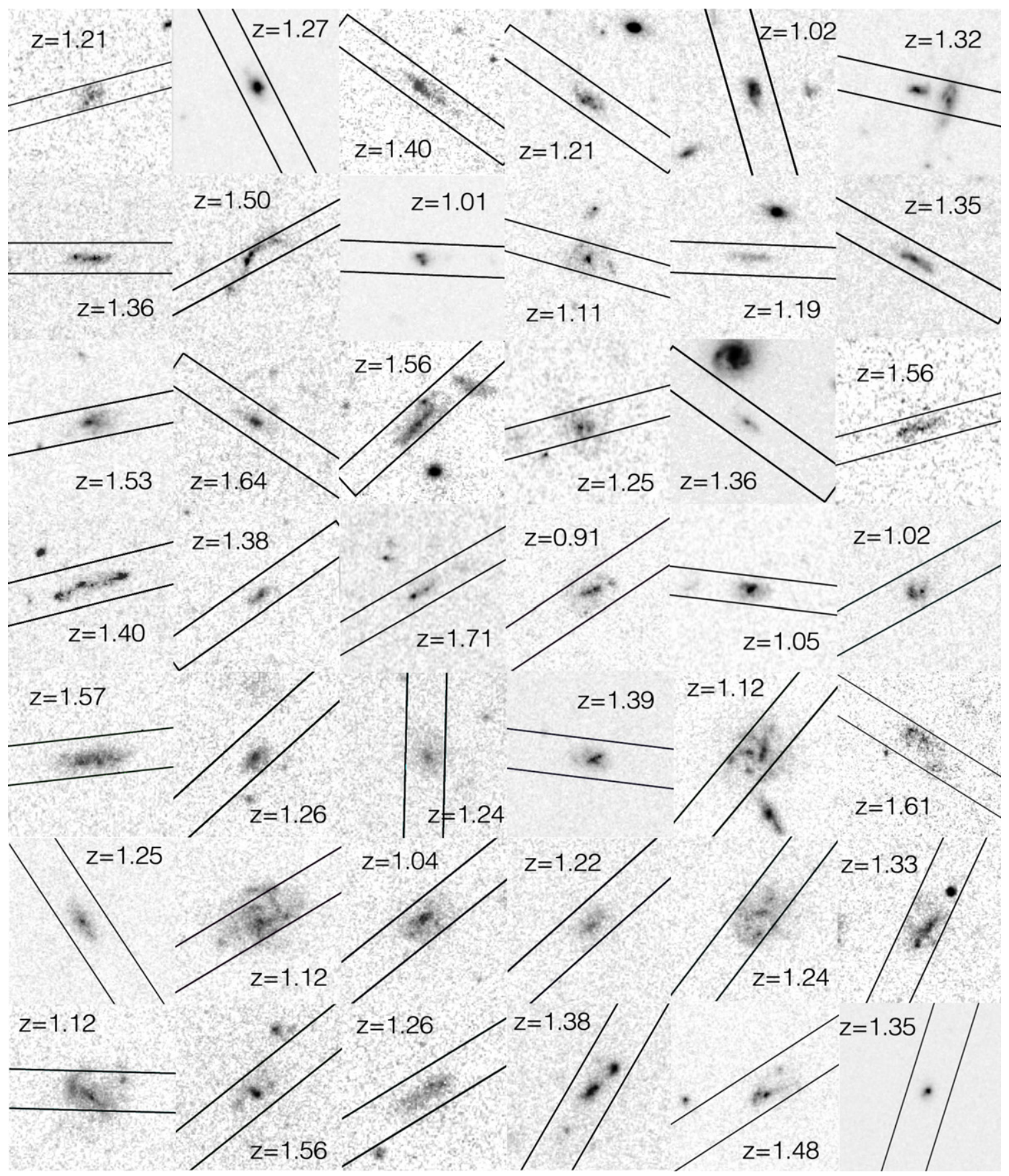

Figure 8. Images of the galaxies in our TF sample, with either the $H S T F 814 \mathrm{~W}$ or $F 850 L P$ and their 1 arcsec slits overlaid. We also include the redshift of each target.

\section{REFERENCES}

Abraham, R. G., Nair, P., McCarthy, P. J., et al. 2007, ApJ, 669, 184 Abraham, R. G., van den Bergh, S., Glazebrook, K., et al. 1997, VizieR Online Data Catalog, 2107, 70001

Bell, E. F., \& de Jong, R. S. 2001, ApJ, 550, 212

Benson, A. 2012, New Astron., 17, 175

Bertin, E., \& Arnouts, S. 1996, A\&AS, 117, 393

Blumenthal, G. R., Faber, S. M., Primack, J. R., \& Rees, M. J. 1984, Nature, 311,517

Bouché, N., Cresci, G., Davies, R., et al. 2007, ApJ, 671, 303

Bournaud, F., Elmegreen, B. G., \& Elmegreen, D. M. 2007, ApJ, 670, 237

Bridge, C. R., Carlberg, R. G., \& Sullivan, M. 2010, ApJ, 709, 1067

Bruzual, G., \& Charlot, S. 2003, MNRAS, 344, 1000

Bundy, K., Ellis, R. S., \& Conselice, C. J. 2005, ApJ, 625, 621

Bundy, K., Ellis, R. S., Conselice, C. J., et al. 2006, ApJ, 651, 120

Bundy, K., Fukugita, M., Ellis, R. S., et al. 2009, ApJ, 697, 1369
Capak, P., Cowie, L. L., Hu, E. M., et al. 2004, AJ, 127, 180

Cassata, P., Cimatti, A., Franceschini, A., et al. 2005, MNRAS, 357, 903 Chabrier, G. 2003, PASP, 115, 763

Chapman, S. C., Scott, D., Windhorst, R. A., et al. 2004, ApJ, 606, 85

Conselice, C. J. 2009, MNRAS, 399, L16

Conselice, C. J., Bundy, K., Ellis, R. S., et al. 2005, ApJ, 628, 160

Conselice, C. J., Rajgor, S., \& Myers, R. 2008, MNRAS, 386, 909

Contini, T., Garilli, B., Le Fèvre, O., et al. 2012, A\&A, 539, A91

Courteau, S. 1997, AJ, 114, 2402

Cresci, G., Hicks, E. K. S., Genzel, R., et al. 2009, ApJ, 697, 115

Davis, M., DEEP Team, \& Extended Groth Strip Collaboration. 2005, BAAS, 37, 1299

Dekel, A., \& Birnboim, Y. 2006, MNRAS, 368, 2

Dutton, A. A., Bosch, F. C. V. D., Faber, S. M., et al. 2011, MNRAS, 410, 1660 Ellis, R. S. 2008, in First Light in the Universe (Saas-Fee Advanced Course 36 , Swiss Society for Astrophysics and Astronomy), ed. A. Loeb, A. Ferrara, \& R. S. Ellis (Berlin: Springer), 259 
Elmegreen, B. G., \& Elmegreen, D. M. 2005, ApJ, 627, 632

Fall, S. M., \& Efstathiou, G. 1980, MNRAS, 193, 189

Flores, H., Hammer, F., Puech, M., Amram, P., \& Balkowski, C. 2006, A\&A, 455,107

Förster Schreiber, N. M., Genzel, R., Bouché, N., et al. 2009, ApJ, 706, 1364

Förster Schreiber, N. M., Genzel, R., Lehnert, M. D., et al. 2006, ApJ, 645, 1062

Förster Schreiber, N. M., Shapley, A. E., Erb, D. K., et al. 2011, ApJ, 731, 65

Genzel, R., Burkert, A., Bouché, N., et al. 2008, ApJ, 687, 59

Genzel, R., Newman, S., Jones, T., et al. 2011, ApJ, 733, 101

Genzel, R., Tacconi, L. J., Eisenhauer, F., et al. 2006, Nature, 442, 786

Giavalisco, M., Ferguson, H. C., Koekemoer, A. M., et al. 2004, ApJ, 600, L93

Gnerucci, A., Marconi, A., Cresci, G., et al. 2011, A\&A, 528, A88

Hammer, F., Flores, H., Elbaz, D., et al. 2005, A\&A, 430, 115

Hopkins, A. M., \& Beacom, J. F. 2006, ApJ, 651, 142

Jogee, S., Miller, S. H., Penner, K., et al. 2009, ApJ, 697, 1971

Jones, T., Ellis, R., Jullo, E., \& Richard, J. 2010, ApJ, 725, L176

Kannappan, S. J., \& Barton, E. J. 2004, AJ, 127, 2694

Kannappan, S. J., Fabricant, D. G., \& Franx, M. 2002, AJ, 123, 2358

Kartaltepe, J. S., Sanders, D. B., Scoville, N. Z., et al. 2007, ApJS, 172, 320

Kassin, S. A., Weiner, B. J., Faber, S. M., et al. 2007, ApJ, 660, L35

Kelson, D. D. 2003, PASP, 115, 688

Koekemoer, A. M., Faber, S. M., Ferguson, H. C., et al. 2011, ApJS, 197, 36

Kron, R. G. 1980, ApJS, 43, 305

Kroupa, P. 2002, Science, 295, 82

Lilly, S. J., Cowie, L. L., \& Gardner, J. P. 1991, ApJ, 369, 79
McCracken, H. J., Capak, P., Salvato, M., et al. 2010, ApJ, 708, 202

Mendes de Oliveira, C., Amram, P., Plana, H., \& Balkowski, C. 2003, AJ, 126, 2635

Miller, S. H., Bundy, K., Sullivan, M., Ellis, R. S., \& Treu, T. 2011, ApJ, 741, 115

Mo, H. J., Mao, S., \& White, S. D. M. 1998, MNRAS, 295, 319

Navarro, J. F., \& Steinmetz, M. 2000, ApJ, 538, 477

Newman, A. B., Ellis, R. S., Treu, T., \& Bundy, K. 2010, ApJ, 717, L103

Oke, J. B., Cohen, J. G., Carr, M., et al. 1995, PASP, 107, 375

Peng, C. 2010, BAAS, 42, 578

Pizagno, J., Prada, F., Weinberg, D. H., et al. 2005, ApJ, 633, 844

Pizagno, J., Prada, F., Weinberg, D. H., et al. 2007, AJ, 134, 945

Puech, M., Flores, H., Hammer, F., et al. 2008, A\&A, 484, 173

Retzlaff, J., Rosati, P., Dickinson, M., et al. 2010, A\&A, 511, A50

Reyes, R., Mandelbaum, R., Gunn, J. E., Pizagno, J., \& Lackner, C. N. 2011, MNRAS, 417, 2347

Rockosi, C., Stover, R., Kibrick, R., et al. 2010, Proc. SPIE, 7735, 77350R

Sales, L. V., Navarro, J. F., Theuns, T., et al. 2011, arXiv:1112.2220

Scoville, N., Abraham, R. G., Aussel, H., et al. 2007, ApJS, 172, 38

Somerville, R. S., Barden, M., Rix, H., et al. 2008, ApJ, 672, 776

Tacconi, L. J., Genzel, R., Neri, R., et al. 2010, Nature, 463, 781

Tutui, Y., \& Sofue, Y. 1997, A\&A, 326, 915

van den Bergh, S., Abraham, R. G., Ellis, R. S., et al. 1996, AJ, 112, 359

Vergani, D., Epinat, B., Contini, T., et al. 2012, A\&A, in press (arXiv:1202.3107)

White, S. D. M., \& Rees, M. J. 1978, MNRAS, 183, 341

Willick, J. A. 1999, ApJ, 516, 47 